\title{
Design of process teaching management system in Vocational Education
}

\author{
QIU Lei, a ${ }^{1,}$ ZHU Tianyu, ${ }^{1, b}$, LIAO Xiaojian ${ }^{1, c}$, HAO Dawei, d \\ ${ }^{1}$ Wuhan Mechanical College, Wuhan 430075, Hubei, China \\ aemail: hnnhlxy@163.com, bemail: 78459906@qq.com, \\ cemail: 276572652@qq.com, 'email: 33736483@qq.com
}

Keywords: Teaching management; Training Guide; Online assessment

\begin{abstract}
Vocational Education in the process of examination and evaluation process, due to the standard was not unified, teacher's subjective factors, the examination results of students were not normative. This paper designed a teaching management system, which was used to guide the student's training, at the same time quantification, determination of test results of the standardization process.
\end{abstract}

\section{System structure}

The teaching management system is divided into four modules: attendance, training guide, online examination and homework exercises. Using the method of credit system, a course is divided into several tasks, when complete a task according to the circumstances to obtain the corresponding credits, the total score of the course is the credits that all the tasks completed by the students. The initial page of the system is the landing page, students enter the student number and password, enter the system.

(1) Attendance

System according to the situation of student login or course tasks automatically complete attendance, the system automatically record the date and the staff task progress, as a formative assessment basis.

After the students enter into the system, select the required course of study, they can see the completed tasks under the course and the credits. Click on the unfinished task to enter the training guide.

(2) Training guidance

In the form of text and video to display course training knowledge points, operating procedures, and so on. According to the guidance students can complete the practical training.

(3) Online assessment

After finish the practical training under the guidance, students can click on the online assessment link to learn just finished training task assessment, to review consolidate knowledge. Under the teacher's weight, the evaluation results can as the credits record in the total credits of the course.

(4) Exercise

According to the student's online examination and misuse, practice varies from person to person; stored in a personal account, facilitate the students to review. After students finish their exercise, Record the operating records into the database, as a formative assessment basis.

After the students complete the task, the teacher can call each student's situation in the database through the administrator terminal, according to the students at ordinary times performance weighted situation, formative assessment result table.

\section{System logical architecture design}

In the design and implementation of network examination system based on $\mathrm{C} / \mathrm{S}$ architecture, involves the user graphical interface design, the TCP/IP protocol, the COM technology, VBA technology, database management system, LAN file transfer and a series of technology. 
Visual Basic (VB )is a visual, object-oriented and event driven windows application development tool launched by Microsoft Corporation. COM(Component Object Module, object component model) is one of the components as a release unit Object model, which makes the software components interact in a unified way. The overall architecture of the system is shown in figure 1.

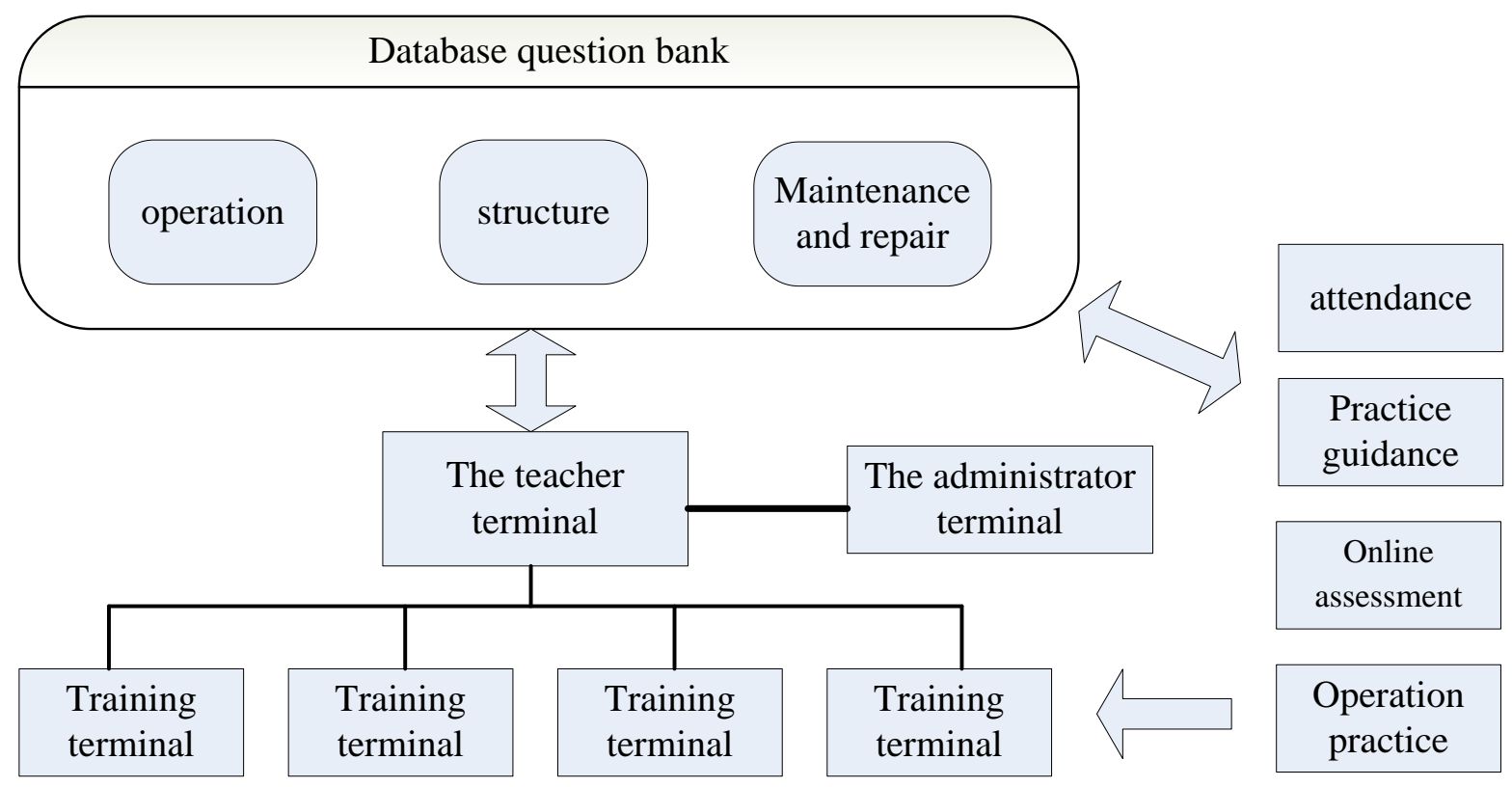

Figure 1 System overall architecture design

\section{Key technologies}

(1) Database technology

Database access using ADO database access technology, in the development process we use SQL programming techniques to perform operations on data in the database. ADO is an abbreviation for ActiveX Data Objects Microsoft, which is the data object that enables the application to access the database. Using ADO data to access control can only use the ADO component to get in touch with the database and operate on the data. Since the ADO object itself uses the ActiveX object, it is very easy to expand on the Internet.

(2) WinSocket programming technology

The basic way of Socket network communication is the request and response mode, the receiver and the sender work collaboratively. Socket programming follows the rules of the packet delivery, each time only to send and receive a packet, to ensure the security and stability of data transmission, but also not too much to occupy system resources. For the ClientSocket component, it can be seen literally, it is used for the requesting party. That is to say, its action is to actively establish a connection. Obviously, the ServerSocket component is used to respond to the side, its action is listening, and passive acceptance of the connection. The property of the ClientSocket component is relatively static, and the relationship between it and the ServerSocket is just the connection and disconnection. And only when the ServerSoeket is accepted, it establishes the connection. The properties of the ServerSocke component are dynamic. With the establishment of a new ClientSoeket connection, it will generate a new Socket corresponding to the ClientSocket, to maintain a separate connection, separate communication. Therefore, in the same ServerSocket can be maintained with multiple ClientSocket and the independent communication at the same time. ServerSocket property Socket.ActiveConnections is used to indicate the number of client connections; the Socket.Connections[Index] attribute is used to access a single ClientSocket connection to the Socket. It is such a structure that makes the WinSocket technology to achieve a stable service program to provide services to multiple clients.

(3) The compression and decompression method of remote screen image's real-time transmission The remote transmission of screen image adopts Client/Server structure, consisting of the Client 
and Server. The client is mainly responsible for the server to send a server to obtain the server screen image request and will be sent from the server to the screen image in real-time display, and the server is responsible for the response of the client's request and capture and send screen images. Because the screen image captured by the server is generally bitmap format, its data is large, if sent directly it will lead to too much network bandwidth, real-time performance, occupy too much system resources and poor stability, so it needs to be compressed before it sent to the client, and client also would like to receive to the screen image data accordingly after decompression can correctly to display screen image. For the image data compression and decompression, the main pursuit is a higher compression rate and faster compression and decompression speed, Delphi has a built-in data stream compression, decompression solution, through the Zlib.pas and Zlibconst.pas two cell file to solve data compression and decompression, to achieve a very high data compression ratio (WinZip), this is a relatively good compression, decompression scheme.

\section{Conclusion}

The development and construction of the course teaching management system in vocational education was an important part of the information construction in colleges and universities. It greatly expanded the regional and the flexibility of the examination. It was an important direction of the development of school examination in the future.

\section{References}

[1] Zhang Quan, Guo Tianjiao. The art of the SQL query.[M].People's Posts and Telecommunications Press, 2014:72 132

[2] Cai Hengwen, Yan Zhenglong, Zhang Li. Principle and application of database system.[M].Nanchang: Jiangxi University Press, 2004:17 74

[3] Liu Changxing. “Sanji”The training network examination system design.[J].China Medical Devices, 2012, 27:28 30

[4] Hao Linyuan. Analysis and design of network training assessment system.[J].RESEARCHES IN MEDICAL EDUCATION,2005, 4:407-409.

[5] Yang Leilei et al. Design and implementation of student status management system based on BS mode.[J]. Science \& Information Technology, 2013, 21:30 31 